\title{
APORTACIONES DE LOS GRUPOS INTERACTIVOS DESDE LA PERSPECTIVA DE ESTUDIANTES Y VOLUNTARIADO
}

\author{
INTERACTIVE GROUPS CONTRIBUTION \\ FROM STUDENTS AND VOLUNTEER'S PERSPECTIVE
}

\author{
Edurne Chocarro de Luis \\ Universidad de La Rioja \\ edurne.chocarro@uniroja.es
}

Sara Mollà Peña

CEIP Caballero de La Rosa. Comunidad de Aprendizaje

sareta0808@gmail.com

\section{RESUMEN}

En este artículo se presenta la definición de grupos interactivos, como una de las actuaciones de éxito propias de Comunidades de Aprendizaje pero, principalmente, su organización y funcionamiento en el Centro Educativo de Infantil y Primaria Caballero de La Rosa de Logroño (La Rioja). Además, se ilustran algunos de los testimonios de sus principales agentes, voluntariados y estudiantes, que sirven de evidencias del impacto que genera el trabajo en grupos interactivos tanto en la mejora del aprendizaje como de la convivencia.

Palabras clave: Grupos interactivos, convivencia, aprendizaje, comunidades de aprendizaje.

\section{ABSTRACT}

This article defines the interactive groups, such as Successful Educational Actions (SEAs) of the Schools as learning communities, mainly its organization and operation within Caballero de la Rosa, a school located in Logroño, La Rioja. Furthermore, it shows some of the testimonials from volunteers and students. All of them serve as evidence of the impact from the work within the interactive groups resulting in the improvement of learning and the relationships within the school.

Key words: Interactive groups, relationships, learning, learning as communities.

\section{INTRODUCCIÓN}

Los grupos interactivos son una de las actuaciones de éxito propias de las escuelas conocidas como Comunidades de Aprendizaje (CdA), aunque pueden llevarse a cabo en cualquier centro. Principalmente, consiste en la resolución de una tarea en grupos de cuatro o cinco estudiantes. Dicho así, podría ser lo propio del aprendizaje colaborativo, pero tiene signos que lo identifican, como la 
participación de una persona externa que modera y dinamiza el trabajo grupal de los estudiantes. Por tanto, su labor no es enseñar sino motivar y certificar la interacción y participación de todos los miembros integrantes. Otra de sus señas de identidad es la heterogeneidad en la división de la clase en grupos más pequeños que se deben mezclar tanto por capacidad, sexo, procedencia etc. Ello exige conocer bien a los estudiantes y analizar con atención el modo de lograr grupos mixtos, no homogéneos, que incluyan a todo el alumnado (INCLUD-ED Consortium, 2011).

Un tercer rasgo es que los grupos interactivos (GGII) se sustentan en las premisas del aprendizaje dialógico (Aubert, Flecha, García, Flecha y Racionero, 2008). Esto es, se aprende desde el diálogo que surge entre sus miembros, pero también con el responsable voluntario.

En definitiva, los grupos interactivos consisten en dividir la clase en grupos heterogéneos de 4 o 5 alumnos y alumnas para la realización de una tarea determinada en un tiempo de unos 15 minutos. Finalizado éste, el alumnado rota a otro grupo donde hay otro voluntario o voluntaria que le propondrá otra actividad. De este modo, el alumnado ha realizado unas cuatro tareas diferentes en una sesión de una hora aumentando así las posibilidades de aprendizaje (Álvarez y Puigdellívol, 2014). Una persona voluntaria (familiar, universitario, agentes comunitarios...) dinamiza cada grupo con el objetivo de que, a través de las interacciones, se aceleren los aprendizajes y se mejoren las relaciones entre el alumnado, produciéndose de este modo lazos de amistad y solidaridad (Elboj y Nielmelä, 2010).

Esta característica hace que el calendario dedicado a grupos interactivos deba estar fijado con previsión para que los voluntarios confirmen su disposición de acuerdo al grupo-clase asignado y ello permite que se desarrollen sin incidencia alguna. Los niños y niñas reciben la visita de personas externas que les acompañarán en su aprendizaje. Principalmente se realiza en las áreas troncales, es decir, matemáticas y lenguaje para poder así incrementar los aprendizajes instrumentales.

Su dinamismo hace que los grupos interactivos permitan lograr una de las máximas educativas como es el principio de la inclusión (Valls y Kyriakides, 2013) ya que garantiza la presencia, participación y protagonismo de todos los estudiantes en el proceso de enseñanza y aprendizaje, tres de los pilares de la educación inclusiva de acuerdo a Echeita y Ainscow (2011), unos de sus principales impulsores. En consecuencia, la propia idiosincrasia de los grupos interactivos se vincula a modos de enseñanza inclusivos (Flecha, 2009; Flecha, García, Gómez y Latorre, 2009), pero si se respetan sus supuestos teóricos previamente explicados derivados del aprendizaje dialógico porque, de otro modo, «el mero hecho de organizar el aula en grupos reducidos no asegura el aprendizaje de todo el alumnado» (Muntaner, Pinya, y De la Iglesia, 2015, p. 150).

Como se ha mencionado, los grupos interactivos se entroncan, aunque no es indispensable, en centros educativos transformados en Comunidad de Aprendizaje (Elboj, Espanta, Flecha, Imbernon, Puigdellívol y Valls, 1998; Díaz-Palomar y Flecha, 2010) que se define como:

Un proceso de transformación social y cultural de un centro educativo y de su entorno para conseguir una sociedad de la información para todas las personas, basada en el aprendizaje dialógico mediante una educación participativa de la sociedad, que se concentra en todos sus espacios, incluida el aula (Elboj, Puigdellívol, Soler y Valls, 2006, p. 74). 
Como indica su definición, este modelo de escuela se fundamenta en la teoría del aprendizaje dialógico (Aubert, Flecha, García, Flecha y Racionero, 2008) que impulsa el poder de las interacciones como motor de aprendizaje pero con la premisa de que éstas deben estar basadas en los principios del aprendizaje dialógico: diálogo igualitario, transformador, solidario, cultural, con creación de sentido e instrumental. Para conocer más sobre su fundamento teórico y fases de transformación se puede acceder a la siguiente página web (http://comunidadesdeaprendizaje.net/).

Los grupos interactivos, como también el resto de actuaciones de éxito propias de Comunidades de Aprendizaje, tienen como «finalidad intensificar el aprendizaje mediante interacciones que se establecen entre todos los participantes (niños/as, profesorado, voluntariado, etc.)» (Flecha y Puigvert, 2002, p. 18) y, además, disminuir los conflictos en el aula (Peirats Chacón y López Marí, 2014; Valls y Kyriakides, 2013).

Para alcanzar estos objetivos y para su buen desarrollo, en los grupos interactivos se exige definir con exactitud los roles de sus agentes participantes (Racionero, García, Aubert y Puigbert, 2009). Así, el tutor o la tutora serán responsables de distintas tareas tanto antes del desarrollo de la práctica como durante su ejecución y finalizada la misma. De este modo, antes de ponerse en marcha, el tutor o tutora planifica los grupos que, como se ha dicho, deberán respetar su heterogeneidad y diseñará las actividades o ejercicios a resolver durante la misma, que serán tantos como grupos establecidos. Las actividades han de estar relacionadas con el currículum que se está impartiendo en el aula en ese momento. Se deben poner actividades de alto nivel de aprendizaje. El papel del tutor durante la sesión es de observación activa. Durante el trascurso, su misión será supervisar el trabajo, vigilar su desarrollo para administrar los tiempos de rotación y, en caso necesario, resolver las dudas de contenidos. También debe intervenir si se observa algún problema de comportamiento en el grupo. Finalizada la clase, será conveniente que dedique un tiempo a valorar el desarrollo de la misma con objeto de reparar en posibles aspectos de mejora en cuanto al propio planteamiento de las tareas, la participación de los estudiantes, interacciones establecidas, etc. Para ello, es importante organizar el horario de modo que permita al profesorado un momento para recoger las aportaciones de los voluntarios.

Por otra parte, la función del voluntariado es dinamizar el trabajo en grupo asegurándose que todos participen y, al mismo tiempo, apoyar el aprendizaje mediante el diálogo pero, en la medida de lo posible, un diálogo entre iguales no sólo de él con los estudiantes. Esto es, éstos ya están familiarizados tanto con el contenido como con la dinámica y por tanto las personas voluntarias no deben suplir la función del maestro, quien ya dedicó su tiempo a enseñar, sino de garantizar que los niños y niñas interaccionen para generar y asentar el aprendizaje. Este matiz es importante. Después, las personas externas deberán ofrecer un feedback de la dinámica cuyos comentarios servirán para mejorar, conocer el trabajo realizado por los estudiantes, reflexionar sobre el diseño de los grupos, etc. No obstante, el mismo compromiso del voluntariado ofrece al centro un valor adicional pues su presencia aporta conocimientos, o capital cultural y posibles sinergias entre la escuela y la comunidad. En definitiva, el alumnado ampliará sus referentes ya que cada persona voluntaria tendrá un modo de hablar, de estar, etc. que servirá de ejemplo para los más pequeños, como luego delatarán en sus testimonios (Fernández Antón, 2014). 
No obstante, lo hasta aquí comentado hace alusión a la ejecución de grupo interactivos en una clase con un grupo-aula pero, su puesta en escena en la totalidad de aulas de un centro educativo, exige una coordinación más exhaustiva para garantizar que todos los grupos-aula dispondrán de tales dinámicas (Ordoñez Sierra, Rodríguez Gallego y Rodríguez Santero, 2017). Con ello se quiere resaltar que el hecho de trabajar con grupos interactivos precisa, a nivel de centro, de una buena planificación de calendario y contar, a ser posible, con un gran número de voluntariado fijo. Esto sería lo ideal, ya que dicha estructura resulta complicada en centros que no reciben tanta ayuda.

No obstante, el esfuerzo de organización y recursos humanos merece la pena dada sus recompensas (Valls y Kyriakides, 2013), como se explicará en el estudio de caso. Una de ellas, ya mencionada, es la mejora de la convivencia ya que, como indica Uruñuela, (2016, p. 23) además de acelerar el aprendizaje:

La educación no solo busca que los alumnos y alumnas adquieran el máximo posible en cuanto a los conocimientos, procedimientos y valores científicos imprescindibles para vivir en la sociedad del siglo XXI. Tiene también como prioridad formar personas que sepan convivir de manera pacífica, que sepan participar en la sociedad compleja que les toca vivir, que sean capaces de organizar sus propias vidas desde sus propios criterios y valores de paz.

Además, son alarmantes las cifras de bullying y cyberbullying entre la juventud (Buelga, Cava, Musitu, y Torralba, 2015; Garaigordobil y Oñederra, 2010) de ahí que la escuela debe priorizar la educación para la convivencia y el respeto. En este sentido el Plan estratégico de convivencia escolar 2016-2020, publicado por el Ministerio de Educación, Cultura y Deporte se apoya en la teoría del aprendizaje dialógico en la resolución de conflictos. Por ello, parece interesante analizar el alcance de los grupos interactivos en este sentido y, para ello, el modo más oportuno es a partir de un estudio de caso en concreto cuyo objetivo es conocer las percepciones de los participantes en grupos interactivos. Por otra parte, el hecho de que un aula cuente con más recursos personales ya es, en sí mismo, un criterio que multiplica las posibilidades de aprendizaje, mejorar la convivencia y ampliar los referentes para los estudiantes.

En el siguiente apartado del texto se explica, inicialmente, la planificación de los grupos interactivos en el Centro Educativo de Infantil y Primaria Caballero de La Rosa y, a continuación, se presentan las percepciones de estudiantes y voluntariado sobre su participación en esta dinámica.

\section{ESTUDIO DE CASO}

\section{Descripción del centro}

El CEIP Caballero de la Rosa situado en Logroño, es el único centro transformado en comunidad de aprendizaje de La Rioja. La inquietud por superar las desigualdades de su alumnado y hacer frente a la diversidad de su procedencia, alentó que el equipo directivo apostara por esta alternativa en el 2012. No obstante, no es una decisión unilateral sino que, de acuerdo al marco teórico, el proceso de transformación debe respetar unas fases siendo la toma de decisión una de 
ellas que supone obtener el consentimiento de la comunidad educativa (Flecha y Puigvert, 2002). Como describen Chocarro y de Lemus (2013), el Caballero de la Rosa alcanzó la aprobación tanto de familiares como del claustro tras superar la fase inicial denominada «sensibilización» que tiene como finalidad familiarizarse con las premisas de este tipo de escuela y, en consecuencia, del aprendizaje dialógico. En esta fase se realizó la formación del profesorado a través de un Proyecto de Innovación Educativa (PIE) contando con la participación de ponentes del CREA y de diferentes CdA que aportaron una visión científica de la educación y consiguieron que toda la comunidad educativa soñara y se ilusionara con una escuela más igualitaria y con mejores resultados académicos.

Establecido como Comunidad de Aprendizaje son muchos los esfuerzos realizados para su asentamiento y establecimiento como tal. Estos esfuerzos han puesto encima de la mesa la buena voluntad de padres y madres así como de la comunidad educativa. Al hilo de ello, la administración dispensó al centro con recursos humanos, esto es, la mitad de la jornada de una maestra está dedicada a la coordinación de la CdA. Dentro de sus funciones está, principalmente, la de organizar y garantizar el funcionamiento de los GGII así como ayudar tanto al profesorado como al voluntariado. Otra de las funciones importantes es la de organizar la formación del profesorado.

Por otra parte, El CEIP Caballero de la Rosa acude a las reuniones de formación del profesorado que organiza en Durango Ikas.kom (red de CdA del País Vasco) y que están organizadas de acuerdo a las siguientes temáticas: grupos interactivos, tertulias literarias dialógicas y prevención de violencia de género. Nuestro centro estuvo acudiendo durante tres cursos a las reuniones de grupos interactivos pero, durante el pasado curso cambió a las de prevención de la violencia de género. La coordinadora asiste a cada reunión acompañada de un miembro del claustro diferente, lo que permite que el profesorado vaya tomando conciencia de la importancia que su formación tiene para la mejora de la aplicación de las actuaciones.

Al equipo directivo le preocupa la inestabilidad de la plantilla de profesorado y considera muy importante la formación del mismo para el correcto funcionamiento de las actuaciones de éxito. Por ello, el curso 2015/2016 todo el claustro participó en un grupo de trabajo en el que se elaboraron actividades para los grupos interactivos. Este pasado curso 2016/2017 se realizó un seminario de formación con textos científicos a través de tertulias dialógicas pedagógicas en las que participaron familiares, además de los miembros del claustro. Se contó con la visita de Rocío García Carrión que ofreció una ponencia sobre "Interacciones como base del aprendizaje en Educación Infantil". Después de escuchar la ponencia, se decidió ampliar a dos horas semanales los grupos interactivos en esta etapa para los próximos cursos. Así también, se intentará mejorar las actividades que se realizan en los grupos poniendo más énfasis a las áreas referidas a la adquisición de la lectura y al razonamiento matemático.

Merece especial atención dedicar unas líneas a explicar la organización de los grupos interactivos en este centro. Concretamente, en el curso 2016/2017 han sido más de 90 las personas que semanalmente acudieron al centro para colaborar. De ellas, la mayoría son estudiantes de grado de la Universidad de La Rioja (UR), aunque otras 4 están cursando un máster y 3 estudiantes de Erasmus, 18 son familiares y 15 son personas ajenas al centro. 
Empezaron realizándose grupos interactivos en todos los cursos de Educación Primaria un día a la semana en las asignaturas de lenguaje y matemáticas y en Educación Infantil una sesión semanal. Se añadió en el curso 2015/2016 un grupo interactivo quincenal en inglés en $5^{\circ}$ de Educación Primaria al que asistieron 2 mamás nigerianas además de una profesora de la universidad y la auxiliar de conversación. En este curso se añadió en el segundo trimestre un grupo interactivo en inglés en $4^{\circ}$ de Primaria que se realizó quincenalmente con la participación de 3 estudiantes chinas de Erasmus, una alumna universitaria y una voluntaria que había participado en otros grupos. Una premisa que seguimos en el colegio es que los familiares no pueden participar en el aula de sus hijos o hijas.

Este elevado número de personas viene motivado por el apoyo de la asociación Ayuda Social Universitaria de La Rioja (ASUR) que opera de puente. Existe una figura de coordinación de ASUR cuyo cometido es, a parte de colaborar en la organización de los grupos, acudir al centro cada día y controlar la asistencia del voluntariado universitario. El coordinador o coordinadora ocupa el lugar del estudiante que no ha podido asistir. De esta manera se cubren casi todas las bajas de forma efectiva.

Al inicio de este curso se realizó un cartel diseñado por una mamá del AMPA en el que aparecía el calendario con los horarios de los GGII de todo el centro, tanto en Educación Infantil como en Primaria y animaba a la gente a participar. La coordinadora organizó al voluntariado según su horario y/o grupo de preferencia. Una vez conocidos los participantes, el equipo directivo organizó una sesión de formación para todos ellos y, establecidas las bases, se dio comienzo a los grupos interactivos en el mes de octubre.

En esta sesión de formación, uno de los aspectos a mejorar suele ser la confusión en el rol del voluntariado. Esto es, consideran que deben explicar contenidos cuando su principal objetivo es dinamizar el grupo. Será el tutor o tutora de clase quien previamente haya explicado la materia y quien resuelva las dudas en el momento. Como explica la responsable de $\mathrm{CdA}$ del centro educativo Caballero de La Rosa:

«En los grupos interactivos el rol del voluntario o voluntaria no es el de explicar, sino el de asegurar las mejores interacciones entre el alumnado para conseguir la realización de la tarea procurando la comprensión de todos los miembros del grupo».

También cabe indicar que uno de los compromisos del voluntariado es ofrecer una valoración del desarrollo de cada sesión de grupos interactivos. Así, al finalizar, las personas se reúnen con el tutor/a de la clase durante 5 minutos y comentan el desarrollo, la participación de los estudiantes o cualquier incidencia que haya podido surgir. El profesorado recoge sus impresiones que le permitirán o bien mejorar tanto el diseño de próximas actividades como de los agrupamientos o, por el contrario, afianzar la propia dinámica si el feedback es positivo. También aprovecha para dar la información sobre el alumnado que considere oportuna para la mejora de su funcionamiento. Para ello, el horario está organizado de tal modo que el tutor o tutora disponga de un momento para atender al voluntariado después de la sesión de GGII. Esta idea consolida la práctica e implica al voluntariado más allá de su mera participación pues su voz en tenida en cuenta para el buen 
funcionamiento. De este modo, se siente parte del mismo pues no solo acude al centro, ayuda y se marcha, sino que participa en el proceso de mejora del proyecto.

A continuación, se presenta el estudio realizado con el objetivo de conocer las percepciones de los participantes en grupos interactivos, tanto voluntariado como alumnado, y así identificar el impacto que genera en ellos o en la convivencia del centro.

\section{METODOLOGÍA}

Se empleó el estudio de casos como diseño cualitativo ya que se ha seleccionado un centro en concreto como unidad de análisis: «el estudio de casos es el estudio de la particularidad y de la complejidad de un caso singular, para llegar a comprender su actividad en circunstancias importantes», señala Stake (1998, p. 11). En concreto, los resultados se extraen de los testimonios de 79 estudiantes de educación primaria y 5 de sus voluntarios. Al finalizar el curso, cada uno escribió su valoración tanto de la dinámica como del rol del voluntariado y, de este modo, se recogió la perspectiva de unos y otros aportando una visión más completa de la misma realidad objeto de estudio, los grupos interactivos. Por otra parte, esta información se completa con las aportaciones del equipo directivo y de la responsable de Comunidades de Aprendizaje del centro, también coautora del texto, quien amablemente relató su testimonio para dar a conocer, de modo personal, el modo de trabajo de este tipo de escuelas y pueda servir de modelos a aquellas personas interesadas. Recogidas sus impresiones, se pasa a comentar los resultados organizados por categorías y acompañados de testimonios que los argumentan. Estas categorías son: convivencia, principio de inclusión, aprendizaje y contribuciones de los voluntarios y, para ello, se ha tomado como modelo el marco teórico de la introducción donde se expusieron estas como beneficios de los grupos interactivo $\mathrm{y}$, por tanto, se ha pretendido comprobar si se hacen evidentes a partir de las aportaciones de sus principales agentes.

No es objetivo medir la frecuencia de respuesta sino extraer algunos de los comentarios e ideas que han sido compartidos por el $100 \%$ de los participantes. De ahí que se haya decidido por presentar aquí algunos testimonios.

\section{RESULTADOS}

\section{Convivencia}

La responsable de CdA indicó dos aspectos para medir la mejora de la convivencia tras implicar al centro en dinámicas que asumen los principios del aprendizaje dialógico, uno de ellos son los partes de amonestación y otro las relaciones durante el tiempo de recreo.

En cuanto a los partes de amonestación (Boletín de Convivencia), en este curso 2016-2017 se ha trabajado en la Comisión de Convivencia el Boletín de Consecuencias, basado en el modelo dialógico de prevención y resolución de conflictos. 
"Recoge el enfoque de Manuel Segura sobre el pensamiento consecuencial y busca la coherencia entre la falta cometida y su reparación material o personal. Por eso hemos buscado que la consecuencia sea reflexiva, enfrentándose a la reacción de los compañeros a través del Club de Valientes y que pidan disculpas por escrito" (Equipo directivo).

El equipo directivo ha evidenciado una disminución de los partes de amonestación en los últimos años y así señala que:

"Concretamente, hemos pasado de un uso de un 30\% a un 3\% y las expulsiones se han reducido desde un $5 \%$ a un $0,5 \%$ ".

En cuanto a los recreos, se ha observado la mejora de la convivencia en varias ocasiones. Así por ejemplo la responsable de CdA cuenta que:

"Un dia, había una alumna de $6^{\circ}$ con problemas de relación, estaba sola en el recreo y se acercaron chicas y chicos de su clase, se sentaron a su lado, hablaron con ella y consiguieron que se uniera al grupo."

También se ha observado que han disminuido el número de peleas físicas.

"Cuando tienen un problema acuden al profesorado, los escuchamos (esto es muy importante, que sientan que vamos a atender a sus problemas) y hacemos que dialoguen entre ellos. Normalmente llegan a un acuerdo. Esto es debido en buena parte a las asambleas de aula en la que se trabajan las emociones y los sentimientos a través de un diálogo igualitario".

\section{Principio de inclusión}

Los grupos interactivos permiten que todo el alumnado tome parte de la clase y, de este modo ningún estudiante queda fuera de la dinámica. En caso de alumnado con necesidades educativas de apoyo específico (ACNEAE), los pedagogos terapeutas acceden al aula los primeros días que se llevan a cabo grupos interactivos pero esta ayuda va desapareciendo conforme se adquiere la dinámica. Esto es, estos estudiantes saben ya cómo trabajar y el resto de miembros de su grupo sabe qué pueden pedir y cuánto les deben ayudar. Así, ellos mismos son parte del trabajo aportando en base a su capacidad. Es interesante observar la colaboración y apoyo en estos casos. La persona responsable de CdA describe que algunos casos han dejado de estar diagnosticados como ACNEAE.

También refuerza que la interacción en grupos interactivos va más allá de la adquisición de vocabulario, también favorece el uso de habilidades sociales, empatía, valores de respecto, etc. A su vez, aquellos niños y niñas más capaces consolidan sus conocimientos al explicar un contenido, como señalan algunos de los más pequeños del centro que incluso ellos, a pesar de su edad, reconocen estas virtudes.

"Los grupos interactivos me han parecido divertidos y buenos para toda la clase porque los que tienen dificultades aprenden de los compañeros y los que lo saben mejor, aprenden a enseñar" (Alumno $5^{\circ}$ E.P.). 


\section{Aprendizaje}

En línea con el anterior punto, todos ellos han manifestado que los grupos interactivos aceleran su aprendizaje y como muestra, algunas de sus apreciaciones:

"No me gustan mucho los grupos interactivos, prefiero el trabajo personal, pero no están tan mal porque repasas lo aprendido y ayudas a tus compañeros” (Alumna $5^{\circ}$ E.P.).

"Me gustan porque es una manera divertida de aprender en grupo. Y si no entiendes algo te pueden ayudar a entenderlo" (Alumna $6^{\circ}$ E.P.).

Estos testimonios ponen encima de la mesa los beneficios de los grupos interactivos en el proceso de aprendizaje:

"Yo desde mi punto de vista me parece que son muy necesarios porque así podemos trabajar en grupo y decir todo lo que creemos, según lo que digamos podemos debatir o estar de acuerdo" (Alumna $6^{\circ}$ E.P.).

\section{Contribuciones de voluntariado}

El hecho de contar con más adultos en clase incrementa, directamente, el número de referentes. Cada una de estas personas externas tiene un modo de ser y comunicarse, además lleva consigo un conocimiento y experiencias únicas. Los estudiantes aprenderán de ellos simplemente por el mero hecho de tener la oportunidad de conocerlos. En definitiva, se enriquecen las opciones de aprendizaje al ser más las posibilidades de interacción.

Por otra parte, y como es lógico, la ratio adulto-estudiantes disminuye al trabajar en grupos interactivos y, en consecuencia, ello ayuda a potenciar el trabajo en clase. A continuación se extraen algunos de los testimonios de los estudiantes:

"Me han ayudado mucho y, por eso, os agradezco mucho. En serio, siempre me he divertido mucho, me ha parecido genial que ayudes a nosotros y a mi”"(Alumna $6^{\circ}$ E.P.).

"Me han gustado los grupos porque me han ayudado a dar un paso al frente y mejorar y creo que por ellos he mejorado. Si pudiera se lo diría a quien me ha ayudado, uno a uno, hora por hora" (Alumno $6^{\circ}$ E.P.).

"Los voluntarios son muy majos y me encantan que dejen su tiempo para estar con nosotros y ayudarnos" (Alumna $3^{\circ}$ E.P.).

Pero el aprendizaje es recíproco ya que también el voluntario se enriquece al participar en esta experiencia, no solo los estudiantes del centro. A continuación se extraen comentarios extraídos de las valoraciones del voluntariado que manifiestan esta idea:

"Gracias por todos los momentos y vivencias inolvidables que hemos compartido con vosotros. Espero que esta familia del Caballero siga creciendo y soñando, todos juntos, como una gran familia”. 
"Al venir al colegio siempre llegaba cansada de la universidad y a veces me cuestionaba si valía la pena o no hacer voluntariado pero, al final de la jornada, siempre tenía la sensación de estar haciendo lo correcto. Creo que ha sido una experiencia muy bonita".

"Ha sido una experiencia maravillosa, He ayudado a muchos niños y lo más importante ellos me han enseñado a mí. Gracias también a los tutores y coordinadores”.

"Ser voluntario acaba siendo una labor muy gratificante sabiendo que ayudas y los niños te lo agradecen. Cuando vas andando por la calle y todos los niños a los que has ayudado, te saludan, abrazan, está genial”.

"Muchas gracias por todo lo que he aprendido y he reflexionado este año. La profesión nunca llega a perfeccionarse. Para ello lo mejor es siempre estar activo (tomando vacaciones, claro) y dialogando".

\section{CONCLUSIÓN}

Este trabajo tiene como objetivo conocer las percepciones de estudiantes y el voluntariado sobre su participación en grupos interactivos y así poder identificar su impacto tanto en el proceso de aprendizaje como en la convivencia de acuerdo con los testimonios recogidos.

Para ello, se realizó una investigación en el Centro Educativo de Infantil y Primaria Caballero de la Rosa, más concretamente se llevó a cabo un estudio de caso. Los principales protagonistas, voluntariado y alumnado, escribieron sus impresiones sobre el tema objeto de estudio, los grupos interactivos, que fueron catalogadas en base a aquellos aspectos considerados beneficios, o impactos, tras trabajar con esta metodología. Estos son: convivencia, principio de inclusión, aprendizaje y voluntariado.

No obstante, primeramente, es reseñable la infraestructura de este centro transformado en Comunidad de Aprendizaje pues el elevado número de personas que colabora en el centro permite que los grupos interactivos se realicen dos veces a la semana en cada grupo de primaria, una hora en la asignatura de lenguaje y otra en matemáticas, y una hora en infantil y, además, se ha extendido a las horas de inglés en dos grupos. Así también es encomiable la organización y buena disposición de familiares y profesorado pues se esfuerzan por llevar al aula los principios del aprendizaje dialógico en todo momento, más allá del proceso de aprendizaje (Aubert, Flecha, García, Flecha y Racionero, 2008). Las interacciones entre todos sus agentes educativos son cercanas, en posición de igualdad y partiendo del supuesto de que todos y todas podemos aprender y aportar de los demás, en todas las actividades y espacios del centro. Todo ello propicia su funcionamiento y son aspectos claves para su buen hacer como la literatura menciona.

Es un hecho real que el centro está ubicado próximo a la universidad y ello permite el trasiego de estudiantes del grado de educación infantil y primaria y de trabajo social que quieren tener un contacto directo con un colegio mediante voluntariado. Más en concreto, mediante la asociación ASUR, estos jóvenes prestan algo de su tiempo para hacer posible los grupos interactivos. Ello propiciará, a su vez, que sean capaces de llevarlo a cabo en el futuro cuando sean ya docentes en ejercicio. 
Como resultados, los testimonios de los estudiantes ponen en evidencia que los grupos interactivos mejoran su aprendizaje. Concretamente, en este centro se observa una mejora de los resultados académicos, lenta pero progresiva. Además, matizan que acelera tanto el proceso de aprendizaje de aquellos que tienen más dificultades como de aquellos más capaces. Los primeros porque se les ofrece una oportunidad para adquirir o consolidar la comprensión a través de las explicaciones de sus compañeros y compañeras. Los segundos, porque al ejercer de profesor, en cuanto que explica al resto la tarea, asienta sus conocimientos, también los refuerzas e incluso puede plantearse dudas que le induce a conocer más. En resumen, las interacciones generadas en grupos interactivos funcionan como motor para el aprendizaje, pero siempre y cuando los grupos están diseñados en cuanto a su heterogeneidad y los voluntarios dinamicen la participación de todos sus miembros (INCLUD-ED Consortium, 2011). Es muy importante incidir en la relevancia que tienen las actividades planteadas ya que deben suponer un reto para los estudiantes. De ahí que la responsable de $\mathrm{CdA}$ de este centro haga hincapié en este aspecto durante el desarrollo de los grupos interactivos. En líneas generales, se debe cuidar los principios básicos de grupos interactivos para que generen los frutos deseados (Muntaner, Pinya y De la Iglesia, 2015).

En cuanto a la convivencia, también mejora al trabajar mediante esta actuación de éxito pues los niños y niñas se conocen entre sí con más cercanía. Al finalizar el curso, han podido trabajar con muchos de sus compañeros y compañeras y han aprendido a prestarse ayuda, a preguntar dudas y a relacionarse (Chocarro y de Lemus, 2013; Ramírez García y Muñoz Fernández, 2012).

Los grupos interactivos también favorecen la inclusión de los estudiantes con necesidad educativa de apoyo específico. Es notable la buena respuesta en este sentido. Los estudiantes aprenden a que cada uno tiene un ritmo de aprendizaje que debe ser respetado.

Finalmente, uno de los impactos de grupos interactivos es el aporte del voluntario en general. Sin ellos, sería imposible llevarse a cabo y, por tanto, acelerar el aprendizaje de este alumnado pero, además, todas estas personas trasladan al aula una mochila cargada de experiencias, conocimiento, vocabulario, expresiones y modos de ser que sirven de referente para ellos. Ellas, a su vez, obtienen ganancias tanto en el ámbito personal y profesional y, además, estrechan más vínculos como la escuela del barrio y ello favorece al clima de convivencia en la calle (Fernández Antón, 2014).

Este trabajo tiene sus limitaciones pero no es el objetivo generalizar estos hallazgos sino presentar la dinámica de un centro que, con tesón y buena voluntad, funciona como Comunidad de Aprendizaje en toda su amplitud, ya que realiza todas las actuaciones de éxito, lo que da una coherencia al proyecto y ha logrado mejorar el clima dentro del centro pero también la relación entre todos los miembros de la comunidad educativa. Los resultados no proceden de un análisis metodológico exhaustivo pero se invitó a participar en un clima de confianza y naturalidad y así recabar sus impresiones reales, sin maquillaje. Siendo así, el centro debe estar orgulloso pues el $100 \%$ de los testimonios plasmaron bondades de los grupos interactivos, solo algunos hicieron alusión a un aspecto de mejora: el ruido que se genera al cambiar de grupo, aspecto fácil de controlar. No obstante, aunque no se pretende extrapolar, las impresiones de los principales protagonistas de grupos interactivos avalan la teoría científica (Elboj, Puigdellívol, Soler y Valls, 2006) y denotan la importancia de aprender a través del diálogo y en colaboración. 


\section{REFERENCIAS BIBLIOGRÁFICAS}

Álvarez, C. y Puigdellívol, I. (2014). Cuando la comunidad entra en la escuela: un estudio de casos sobre los G.I., valorados por sus protagonistas. Profesorado. Revista de Currículum y Formación del Profesorado, 18(3), 239-253.

Aubert, A., Flecha, A., García, C., Flecha, R. y Racionero, S. (2008). Aprendizaje dialógico en la sociedad de la información. Barcelona: Hipatia Editorial.

Buelga, S., Cava, M., Musitu, G. y Torralba, E. (2015). Cyberbullying aggressors among Spanish secondary education students: an exploratory study. Interactive Technology and Smart Education, 12 (2), 100-115. DOI: https://doi.org/10.1108/ITSE-08-2014-0025

Chocarro, E. y de Lemus, C. (2013). Impresiones sobre la evidencia de una práctica inclusiva mediante grupos interactivos. Berceo, 165, 205-222.

Díez-Palomar, J. y Flecha García, R. (2010). Comunidades de Aprendizaje: un proyecto de transformación social y educativa Revista Interuniversitaria de Formación del Profesorado, 24, 1, 19-30.

Echeita, G. y Ainscow, M. (2011). La educación inclusiva como derecho: marco de referencia y pautas de acción para el desarrollo de una revolución pendiente. Tejuelo: Revista de Didáctica de la Lengua y la Literatura, 12, 26-46.

Elboj, C., Espanta, M., Flecha, R., Imbernon, F., Puigdellívol y Valls, R. (1998). Comunidades de aprendizaje: sociedad de la información para todos (cambios sociales y algunas propuestas educativas). Contextos Educativos, 1, 53-75.

Elboj, C., Puigdellívol, I., Soler, M. y Valls, R. (2006). Comunidades de aprendizaje. Transformar la educación. Barcelona: Graó.

Fernández Antón, E. (2014). La inteligencia cultural en los G.I.: un estudio de caso en la Comunidad de Aprendizaje La Pradera de Valsaín (Segovia). Aloma, 32(2),

Flecha, R. (2009). Cambio, inclusión y calidad en las comunidades de aprendizaje. Cultura y Educación, 21(2), 157-169. DOI: https://doi.org/10.1174/113564009788345835

Flecha, A., García, R., Gómez, A., y Latorre, A. (2009). Participación en escuelas de éxito: una investigación comunicativa del proyecto Includ-ed. Cultura y Educación, 21(2), 183-196. DOI: https://doi.org/10.1174/113564009788345899

Flecha, R. y Puigvert, L. (2002). Las comunidades de aprendizaje: una apuesta por la igualdad educativa. REXE, 1, 11-20.

Garaigordobil, M. y Oñederra, J. A. (2010) La violencia entre iguales: Revisión Teórica y estrategias de intervención. Madrid: Pirámide.

García-Carrión, R., y Díez-Palomar, J. (2015). Learning communities: Pathways for educational success and social transformation through interactive groups in mathematics. European Educational. Research Journal, 14(2), 151-166. DOI: https://doi.org/10.1177/1474904115571793

INCLUD-ED Consortium (2011). Actuaciones de éxito en las escuelas europeas [colección Estudios CREADE, n. ${ }^{\circ}$ ]. Madrid: Secretaría General Técnica. Ministerio de Educación.

Muntaner, J. J. Pinya, C. y De la Iglesia, B. (2015). Evaluación de los grupos interactivos desde el paradigma de la educación inclusiva. Revista Electrónica Interuniversitaria de Formación del Profesorado, 18(1), 141-159. DOI: https://doi.org/10.6018/reifop.18.1.214371 
Ordóñez Sierra, R., Rodríguez Gallego, M. y Rodríguez Santero, J. (2017). Grupos interactivos como estrategia para la mejora educativa: estudio de casos en una comunidad de aprendizaje. Revista de Investigación Educativa, 35(1), 71-91 DOI: https://doi.org/10.6018/rie.35.1.247061

Peirats Chacón, J., y López Marí, M. (2014). Los grupos interactivos como estrategia didáctica en la atención a la diversidad [Interactive groups as strategy teaching in the attention to diversity]. ENSAYOS. Revista de la Facultad de Educación de Albacete, (28), 97-211

Racionero, S., García, R., Aubert, A. y Puigbert, L. (2009). Los modelos sociales de enseñanza: los grupos interactivos. Competencias básicas y modelos de enseñanza escuela, 4, 1-8.

Ramírez García, A., y Muñoz Fernández, M. C. (2012). Prácticas inclusivas de los docentes en la convivencia escolar y en la organización y funcionamiento de los centros de educación primaria de la zona norte de Córdoba. Revista de Investigación Educativa, 30(1), 197-222.

Stake, R. E. (1998). Investigación con estudio de casos. Madrid: Morata.

Uruñuela, P. M. (2016). Trabajar la convivencia en los centros educativos: una mirada al bosque de la convivencia. Madrid: Narcea.

Valls, R y Kyriakides, L. (2013). The power of Interactive Groups: how diversity of adults volunteering in classroom groups can promote inclusion and success for children of vulnerable minority ethnic populations, Cambridge Journal of Education, 43(1), 17-33, DOI: https:// doi.org/10.1080/0305764X.2012.749213 